\title{
To affinity and beyond
}

\author{
Antibodies are the researcher's Swiss army knife: there's a tool for every purpose, and \\ they're made in more shapes and sizes than ever. Pete Moore and Julie Clayton report.
}

\begin{abstract}
A ntibodies not only protect us from infection, they have been exploited for years in the laboratory - in diagnostic tests, to purify proteins and as the workhorses of cell biology to detect, locate and identify cellular proteins. Designed by evolution to recognize and bind virtually any molecule that could exist, what more could we ask of them? Quite a lot, it seems. To meet the demands of today's researchers and drug developers, antibodies are being cut down to size, tweaked into new shapes, and manufactured in entirely new ways.

Antibodies as research reagents are ubiquitous. Therapeutic antibodies have had a bumpier ride, but now they're back — and here to stay. Crucially, the large pharmaceutical companies have regained interest. "Antibodies are causing a lot of excitement because there are not many new drugs out there and $30 \%$ of the biotech industry is driven by antibody technology," says Peter Hudson of CSIRO Health Sciences and Nutrition in Melbourne, Australia. Greg Winter of the MRC Laboratory of Molecular Biology in Cambridge, UK, and co-founder of antibody drug-discovery company Domantis, agrees. "A year or two back we were interested in both clinical and research applications, but it is very difficult to make money from research applications and it is clear that the funding
\end{abstract}

from investors requires us to have a pretty clear clinical focus."

Simple financial incentives drive much of the work in therapeutic antibodies. Donald Drakeman, president and chief executive of Medarex in Princeton, New Jersey, estimates that it takes some US\$20 million to get a traditional small-molecule drug ready for clinical testing, whereas this could cost only $\$ 2$ million for an antibody.

\section{Consulting the library}

The business end of an antibody molecule is the antigen-binding site. This is the part of the antibody that latches onto a specific antigenic determinant or epitope - a complementary structure on the surface of another molecule. The antigen-binding site is also known as the variable region, or variable domain, as it is the part of the antibody molecule that varies most among the billions of different antibodies that can be made, either naturally in the human body or by protein engineering. As a large biological molecule such as a protein can contain many epitopes, you can either produce monoclonal antibodies that target just one epitope on a molecule, or raise a collection of antibodies — polyclonal antibodies that bind to a number of different epitopes on the molecule.
For therapeutic use in humans, the gold standard is a fully human monoclonal antibody. Antibodies in which the antigen-binding site is from a mouse, even when most of the rest of the antibody is human, as in chimaeric and humanized antibodies, will always risk triggering an unwanted immune response that, at best, neutralizes the therapeutic antibody and renders it ineffective. Finding the right human antibody has been made much simpler by the advent of genetic engineering and the development of vast human antibody libraries packed with potential candidates. These libraries are based on phage-display technology and store and express billions of human variable regions. Each phage carries a DNA sequence encoding a different variable region, and displays the corresponding protein on its surface, usually as part of a phage coat protein. When a potential drug target is screened against the library, only phage carrying antibodies with an affinity for the target lock on to it. The captured phage are multiplied in bacteria, and the antibody genes re-isolated, to be fashioned into genes that can be used to produce large amounts of fully functional monoclonal antibodies in mammalian cell cultures, or into smaller antibody fragments that can be produced more cheaply in yeast or bacterial culture.

\section{PLAYING WITH THE PIECES}

Regeneron of Tarryton, New York, has turned the usual way of using antibodies as therapeutics on its head. To produce therapeutic agents that will mop up unwanted cytokines or growth factors, the company has developed a hybrid molecule called a 'Trap'. This is made from the stem of an antibody fused to two receptors for the target molecule. In this

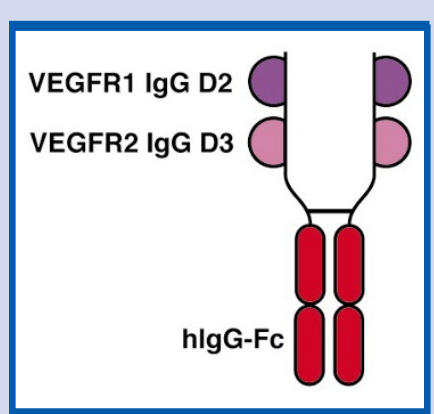

Regeneron's VEGF Trap. application, the antibody stem gives the molecule a long half-life in the circulation, while the receptors replace the variable regions. Traps therefore bind tightly to their target and can be given at infrequent intervals. Regeneron has created an interleukin-1 (IL-1) Trap that it hopes may be effective against rheumatoid arthritis, and is developing a dual IL-4/IL-13 Trap as a potential treatment for asthma and other allergies. A Trap for vascular endothelial growth factor (VEGF) is in clinical trials for the treatment of cancer by preventing the growth of blood vessels into solid tumours and non-Hodgkin's lymphoma. "Every time we compare them with a conventional antibody they have affinities two or three orders of magnitude higher," says George Yancopoulos, Regeneron's chief scientific officer. Unlike antibodies, Traps will pick up every molecule that normally binds to the receptor, so the VEGF Trap will also bind the related growth factor PLGF.

Immunotoxins - antibodies linked to cytotoxic drugs - such as Wyeth's Mylotarg, have the potential to avoid some of the toxic side effects of traditional cancer chemotherapy by delivering the drug directly to the tumour cell. Seattle Genetics of Washington state is developing SGN-15, an antibody-drug conjugate composed of a mouse-human chimaeric monoclonal antibody chemically linked to the cytotoxic drug doxorubicin. This is currently in a phase II clinical trial for the treatment of lung cancer. The antibody binds to a Lewis'-related antigen expressed on many types of solid tumour. SGN-35, one of the company's second-generation immunotoxins now in preclinical trials, incorporates a potent synthetic drug and a novel chemical linkage that makes the molecule more stable in the blood. 
A recent success of the phage-display technology is HUMIRA, the first fully human monoclonal antibody to be approved for therapy, developed by Cambridge Antibody Technology (CAT) in Cambridge, UK, and Abbott Laboratories in Abbott Park, Illinois. This antibody, which blocks the inflammatory cytokine TNF- $\alpha$, was approved in the United States in 2002 and in Europe in September this year as a treatment for the symptoms of rheumatoid arthritis.

CAT's phage-display library holds singlechain variable fragments $(\mathrm{scFv})$ which consist of the variable ends of a light and a heavy chain linked with a short peptide. The library now boasts more than 100 billion distinct antigen-binding specificities. And in the world of antibody discovery, most people think that size matters. The larger the library, the greater the chance that it contains an antibody for your given target. "It means you can get reasonably high-affinity antibodies out of your first collection," says Winter.

The library started as a collection of antibody genes corresponding to antibodies

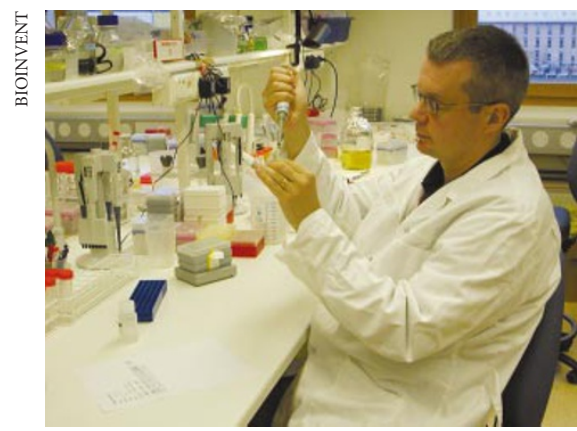

Eskil Söderlind: 10 billion antibodies. found in the blood of healthy people, but CAT has shuffled the DNA sequences that code for the variable region to give even more different types of antigen-binding site. Having started with human genes, CAT believes its antibodies are less likely to create immunogenic responses than libraries built with synthetic sequences.

Antibody discovery and production company BioInvent in Lund, Sweden, has a similar library called n-CoDeR, which holds human scFv. Like CAT, BioInvent starts with natural human antibody genes as the source of the DNA sequences, which are then further diversified by making variants of the antigen-binding regions. "We have taken evolution beyond nature and the library now has more than $10^{10}$ members," says Eskil Söderlind, who invented the n-CoDeR technology with Carl Borrebaeck. BioInvent can screen up to 20,000 clones per day against a customer's target antigen on its automated system. The scFv fragment genes are then converted into complete antibody genes, and the antibody itself produced in larger quantities from genetically engineered mammalian cells.

The biopharmaceutical company Dyax in Cambridge, Massachusetts, has phage-display libraries holding Fab fragments - an entire single 'arm' of an antibody. Although the company holds many of the foundation patents for phage-display technology, Dyax is a relative newcomer in applying phage display to antibodies, having initially focused on peptide and protein display. The company has moved rapidly into the antibody arena by cross-licensing its intellectual property with other major players, such as Genentech, Xoma, Affimed, Biosite and CAT. Chief scientific officer Clive Wood believes that one of the company's strengths is the newness of its antibody libraries. "We are the new kid on the block, but because of that we have important advantages. We have been able to incorporate new features into our Fab library, such as having a mixture of natural and synthetic diversity that allows us to pull out antibodies with picomolar to nanomolar affinity." The largest Dyax library contains about 37 billion distinct human antibodies, and the company has two therapeutic proteins derived from phage display in three phase II trials.

The Human Combinatorial Antibody Library (HuCAL) of MorphoSys in Martinsried, Germany, offers 10 billion totally synthetic variable regions modelled on partial human variable regions and displayed in phage. MorphoSys has now introduced HuCAL GOLD, in which a disulphide bond

\section{GOING INTO PRODUCTION}

"Some of the therapeutic antibodies are required now in tens of thousands of grams per year," says John Birch, chief scientific officer of the Lonza Group, based in Basel, Switzerland, which is planning three new 20,000-litre mammalian cell-culture reactors in the United States for monoclonal antibody production, in addition to its present capacity. Antibody harvests from cultured mammalian cells are around a gram per litre of culture.

But the cost of scaling-up production using mammalian cells can be prohibitive, particularly for a clinical trial of an antibody that may not turn out to be worth it. Looking to fill a gap in the market are alternatives such as the transgenic goats of GTC Biotherapeutics in Framingham, Massachusetts, and the transgenic rabbits produced under a GTC Biotherapeutics licence by BioProtein Technologies of Paris, France. "If I need it, I breed it - the walls of my bioreactor are expandable," says Thomas Newberry, vice-president of GTC Biotherapeutics. He estimates that the cost of producing a founder goat is about US $\$ 10$ million. Although pricey, this is a fraction of the estimated \$200 million-500 million that is needed to build a large-scale mammalian cellculture bioreactor. The company estimates that a single goat can produce up to a kilogram of pure antibody per year. Goat-produced antibodies are in clinical trials to determine whether they match the safety, efficacy and pharmacokinetics of more traditionally produced counterparts.
"There's a whole category of antibodies that have not been effectively explored for therapeutic or preventive use, and for facing down viruses in the mouth, genito-urinary and respiratory tract," says Elliott Fineman, president and chief executive of Planet Biotechnology in Hayward, California. These are the secretory IgA antibodies, which cannot be produced from mammalian cell culture in significant amounts. Instead, his company is using transgenic tobacco plants to produce CaroRx, a monoclonal secretory IgA against the bacterium Streptococcus mutans, a leading cause of tooth decay. Originally created as 'Guys 13' by Thomas Lehner and Julian Ma at Guy's Hospital in London, CaroRx has shown its ability to block colonization by $S$. mutans in phase II clinical trials, and Planet Biotechnology is hoping to apply shortly for a licence to market the antibody in Europe for topical application by dentists and patients following antiseptic cleansing of teeth. If the company succeeds, it will be the first 'plantibody' to reach the market.

Fineman estimates that building, equipping and validating a facility to produce 100 kilograms of antibody from plants would cost between $\$ 30$ million and \$50 million. Keeping cost down is particularly important for antibodies intended for non-therapeutic use against diseases that are not life-threatening. "Nobody's going to take a £2 hike in the cost of toothpaste," says Ma. 
links the antibody to the phage instead of the antibody being an integral part of one of the coat proteins. This makes it easier to separate the antibody at a later stage. MorphoSys also offers 'Antibodies by design', a service that will develop recombinant monoclonal HuCAL antibodies for individual targets. "This is especially interesting for researchers in industry and academia, as the turnaround time is only about two months and the cost starts at about E5,000 [US\$6,000]," says Claudia Gutjahr-Loeser of MorphoSys.

Rather than going for comprehensiveness, a human-antibody library can be biased in favour of particular targets, for example by taking the initial antibody coding sequences from individuals vaccinated against a particular disease. Blood from soldiers immunized against anthrax was the starting point for a Fab antibody library that has allowed Katherine Bowdish and her

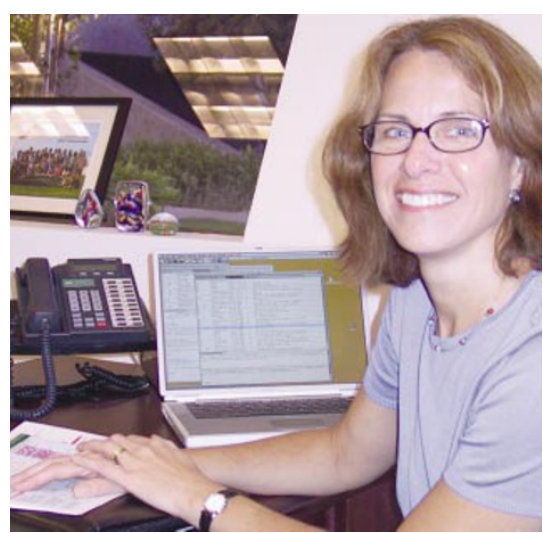

Katherine Bowdish: getting to grips with anthrax. colleagues at Alexion Antibody Technologies in San Diego, California, to identify highaffinity human monoclonal antibodies to critical elements of the anthrax toxin (M. A. Wild et al. Nature Biotechnol. 21, 1305-1306; 2003). The technology is also applicable to diseases such as smallpox, says Bowdish.

As the source of its library, Affitech in Oslo, Norway, also uses blood from selected donors known to produce antibodies of interest, such as cancer patients, or people who have been vaccinated or are recovering from an infectious disease. Affitech's chief executive Martin Welschof says that this, combined with the company's proprietary screening system, avoids the need for repetitive rounds of selection and means that it can move from a $10-\mathrm{ml}$ blood sample to a highaffinity human monoclonal antibody of the desired specificity within four weeks.

\section{Small is beautiful}

If a therapeutic antibody has to penetrate far into body tissues, or into a tumour, for example, smaller may well be better. Although some companies are concentrating on scFv antibody fragments, UK-based Domantis is producing even smaller human antibodies from its phage libraries: these consist of just a single variable domain.

Like scFv fragments, these 'domain antibodies' can be mass-produced relatively cheaply in Escherichia coli or yeast. They are also very stable, which opens up new options for therapy, such as a powder that can be inhaled to treat asthma and other lung diseases, or a tablet to be taken orally for gut inflammation. "We're only going after areas where domain antibodies have unique

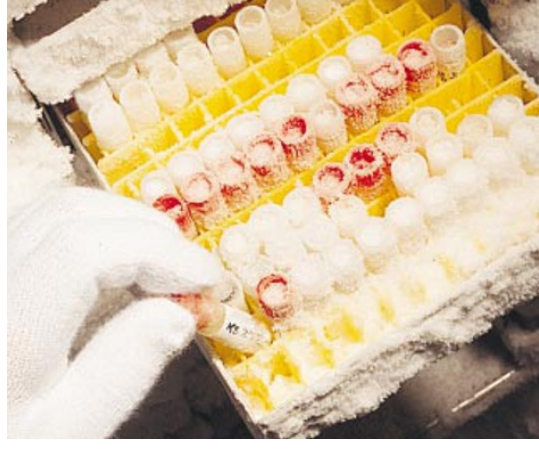

Frozen phage: CAT's library in store.

advantages," says Ian Tomlinson, co-founder and chief scientific officer of Domantis. In partnership with Peptech in North Ryde, New South Wales, Australia, Domantis is developing a domain antibody against TNF$\alpha$ for the treatment of rheumatoid arthritis and other inflammatory diseases.

Part of the inspiration for pursuing domain antibodies was the natural domain antibodies found in camels, dromedaries, llamas - and sharks. These natural antibodies are also being explored for their potential applications by start-up biopharmaceutical company Ablynx of Zwijnaarde, Belgium.

\section{Mouse power}

Both Abgenix of Fremont, California, and Medarex of Princeton, New Jersey, take a different approach to making large numbers of different human antibodies for screening - they get mice to do it for them. In Abgenix's XenoMouse and Medarex's HuMab-Mouse, murine germ-line antibody genes have been replaced with human ones.

\section{ANTIBODIES WHERE YOU WANT THEM}

Antibodies are normally built inside cells, take up their final form as they are secreted from the cell, and function exclusively outside cells, binding to cell surfaces and extracellular molecules. But Terry Rabbitts at the MRC Laboratory of Molecular Biology in Cambridge, UK, wants to use antibodies' highly specific binding properties inside the cell itself. He has engineered cells to make fragments consisting of just a heavy-chain variable domain and hold them within the cell. These single-domain antibodies can interact with intracellular proteins at binding affinities of 10 nanomolar or better. At Iclectus, an MRC spin-off company based in London, Rabbitts' colleagues are developing these fragments as intracellular antibodies or 'intrabodies'.

They became interested in intrabodies while they were unravelling the role of transcription factors involved in producing chromosomal translocations in tumour cells. Using intrabodies to bind and block these transcription factors inside the cell opened up a new range of research possibilities as well as pointing the way to new anticancer therapies. Transcription factors operate by binding other proteins, so "if you have an

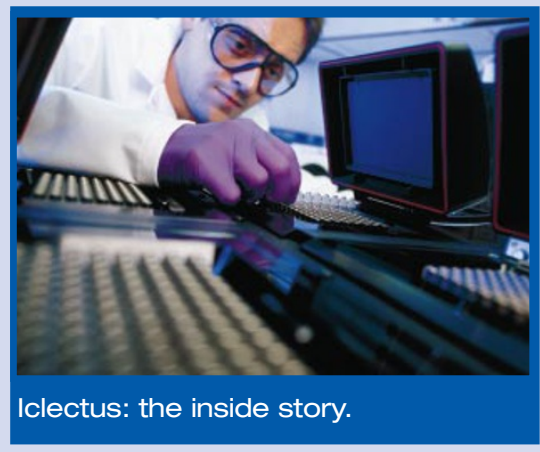

intrabody that has an affinity close to or better than the affinity of the interacting partner, you should be able to block the interaction using the intrabody", says Rabbitts.

Intrabodies offer the cell biologist the prospect of highly specific tools that can probe protein-protein interactions in situ more delicately than techniques that simply remove one of the proteins from the scene by antisense or gene knockout. Because an intrabody binds to a specific site on a protein, it can block a protein's interaction with one partner while allowing it to continue acting with another.

Another use is for the validation of potential drug targets, one of the first and vital steps in drug discovery. Most potential target proteins are modular, so intrabodies that bind to and block the function of individual modules could help to determine which part of the protein is key to causing the disease.

Placing localization signals on an intrabody allows it to be directed to various parts of the cell, such as the nucleus. "With intrabodies you can be more versatile in your experiments and ask more questions than, say, with RNA interference," says Rabbitts. 
When challenged by a foreign protein, these mice produce fully human, functional antibodies. Medarex markets the HuMAb-Mouse as one part of its UltiMAb platform. "Buying the UltiMAb system allows our partners to use any of three different mice to develop products," explains Drakeman. One is the HuMAb-Mouse. The second is Kirin TC Mouse, initially built by Kirin Brewery in Tokyo, Japan. This is a transchromosomal mouse containing the 15-megabase segment of human chromosome 14 that contains all the genes needed to build human antibodies. The third is KMMouse, a cross-bred version of HuMAb-Mouse and KM-Mouse. Using this technology, Medarex has generated 15 antibodies that are currently in clinical trials, while Abgenix has two in its own pipeline and others being developed by Pfizer and Amgen.

Whether you use a phage or mouse system can be influenced by what you are trying to achieve. Engineered mice don't have all the answers, claims BioInvent's Söderlind. "Mice won't react with all substances, as they may have innate tolerance to the antigen, and if the target is toxic they may die before any antibodies are formed," he says. In those circumstances, in vitro phage-based systems offer a good way forward. On the other hand, say the owners of engineered mice, phage libraries can only display antibodies that do not kill bacteria.

\section{Antibody assays}

Despite a preference for human antibodies in the clinic, they are not the first choice in other settings. Monoclonal antibody technology started with mouse antibodies, and many people argue that mice are still in the driving seat. Winter recalls remarking to the late César Milstein, who created mouse monoclonal technology in the 1970s, that phage display was going to bypass the whole business of immunizing animals to get monoclonal antibodies. "He bridled at this a bit and said 'you are wrong' — and I think he was right," says Winter. Milstein believed there would be a niche for all types of antibodies. And some of those niches are huge.

For when antibodies are being used as detection reagents in the laboratory, there is no need for expensive high-tech human antibodies. Rat, mouse and hamster monoclonal antibodies derived by well-established hybridoma technology, as well as polyclonal antibodies raised by immunizing larger animals, are the mainstay of laboratory immunoassays and immunodetection techniques such as ELISA (enzyme-linked immunosorbent assay) and its variant ELISpot, western blotting, flow cytometry and immunofluorescent staining of cells and tissues.

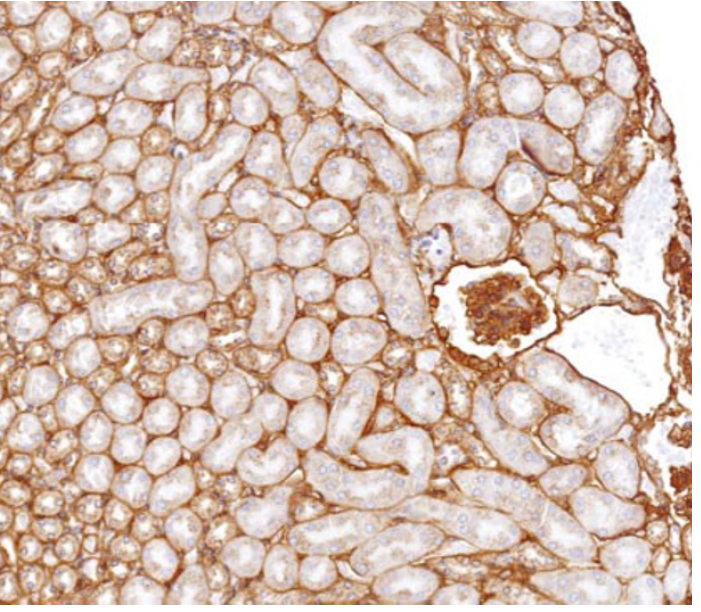

which works really well," says Andrew Bradbury of the Los Alamos National Laboratory in New Mexico, who is looking to commercialize the technology.

But despite the prospect of novel reagents such as fluorobodies and intrabodies, polyclonal antibodies raised in the traditional way by immunizing animals are still in demand, even for the latest protein array technologies. For example, using highthroughput techniques, Eurogentec in Seraing, Belgium, will take a customer's mRNA library and produce peptides representing different parts of the encoded proteins. Polyclonal antibodies specific for each peptide are then raised in rabbits. After selection to reduce the chance of crossreactions,

Bender MedSystems of Vienna, Austria, offer 'instant ELISAs', complete ELISA kits to which the user only has to add water. These are available for a range of human, mouse and monkey immune-system cytokines and other proteins. Arrays of hundreds of antibodies are also currently the basis for the up-and-coming field of 'protein chips', the proteomics equivalent of DNA microarrays (see Nature 424, 581-587; 2003).

Companies such as Sigma-Aldrich in St Louis, Missouri; Chemicon in Temecula, California; Active Motif in Carlsbad, California; MabTech in Nacka Strand, Sweden; and Progen Biotechnik in Heidelberg, Germany, are just some of many that supply off-theshelf monoclonal and polyclonal antibodies against thousands of cellular targets.

Interest doesn't stop at antibodies against human and mouse proteins for biomedical research. BD Biosciences Pharmingen has thought it worthwhile to screen its large catalogue of mouse monoclonal antibodies to human intracellular structural and signalling proteins for good crossreactivity against the homologous Drosophila proteins. It now offers a list of about 25 high-quality monoclonal antibodies selected to give good results in Drosophila, and is adding more.

This wide array of laboratory reagents may soon be joined by a useful molecule that combines the binding specificity of an antibody with built-in fluorescence. These 'fluorobodies' would circumvent the need to add fluorescent tags to conventional antibodies. Fluorobodies consist of a super-folding version of green fluorescent protein (GFP) with the minimal antigen-binding portions of antibodies, conferring the specific protein-binding ability, grafted into four loops at one end of the GFP. They function as well as conventional antibodies in ELISAs, gel-shift assays, protein chip and flow cytometry assays - and without the need for secondary antibodies to provide a signal. "It's a once in a lifetime invention the antibodies are spotted onto glass slides to produce a customized array of up to 1,000 antibody specificities, which can be used to screen and compare tissue samples for the differential expression of multiple proteins. The approach is intended to complement the ELISA, which screens a single antibody against multiple cell or tissue samples, according to chief executive Gottfried Proess.

\section{Antibody's-eye view}

A renaissance is taking place in cellular pathology, according to Tony Warford of the Wellcome Trust's Sanger Institute in Cambridge, UK. This is thanks to the development of automated high-throughput immunohistochemistry of intact normal or diseased tissue samples. Labelled antibodies are used to stain tissues to identify the expression of particular proteins. As a senior project leader for John McCafferty's 'Atlas of Gene Expression' at the Sanger Institute, Warford, is pioneering the linking together of automated immunohistochemistry techniques.

By applying phage-produced monoclonal antibodies as probes to composite microarrays of tissue samples, he can see which proteins are expressed, when and where, in a given cell and tissue type. The tissue microarrays, which are created using automated technology from Beecher Instruments in Sun Prairie, Wisconsin, and Chemicon in Ternecula, California, consist of paraffin wax-embedded blocks of human or animal tissues containing up to 500 individual minitissue samples (each about $0.6 \mathrm{~mm}$ across and $3 \mathrm{~mm}$ deep). These can then be sliced up into around 100 individual sections for immunohistochemical staining.

With automated processing, it will be possible to screen and interpret up to 20,000 individual samples — all in a day's work. Pete Moore is a freelance science writer based near Bristol, UK. Julie Clayton is a science writer based in Bristol, UK. 